\title{
CONVEXITY CONDITIONS RELATED WITH 1/2 ESTIMATE IN BOUNDARY PROBLEMS WITH SIMPLE CHARACTERISTICS. II
}

\author{
MASATAKE KURANISHI
}

Choose a submanifold (not necessarily closed) $\mathscr{N}^{2}$ of $S^{*} U$, which is transversal to $C^{\lambda}$ and intersects $C^{\lambda}$ only at $\left(x^{0}, \zeta^{\lambda}\left(x^{0}\right)\right)$. Pick a nonzero $u$ in $W^{\lambda}\left(x^{0}, \zeta^{\lambda}\left(x^{0}\right)\right)=$ the image of $\rho_{1}^{\lambda}\left(x^{0}, \zeta^{2}\left(x^{0}\right)\right)$. Then the function

$$
f_{u}(x, \xi)=\left|a(x, \xi) \rho_{1}^{\lambda}(x, \xi) u\right|^{2}
$$

viewed as a function on $\mathscr{N}^{2}$ is of class $C^{\infty}$ and nonnegative, and $\left(x^{0}, \zeta^{2}\left(x^{0}\right)\right)$ is its isolated zero, i.e., an isolated critical point of $f_{u}$ on $\mathscr{N}^{2}$.

Definition 2.3. Assume that the characteristics of $A$ is smooth. We say that a characteristic $\left(x^{0}, \zeta^{2}\left(x^{0}\right)\right)$ of $A$ is nondegenerate if and only if $\left(x^{0}, \zeta^{2}\left(x^{0}\right)\right)$ is a nondegenerate critical point of $f_{u}$ on $\mathscr{N}^{2}$ for all nonzero $u$ in $W^{\lambda}\left(x^{0}, \zeta^{\lambda}\left(x^{0}\right)\right)$. We say that the characteristics of $A$ are nondegenerate when each characteristic is so.

Since $f_{u}$ on $\mathscr{N}^{2}$ takes the minimum value at $\left(x^{0}, \zeta^{2}\left(x^{0}\right)\right)$, the above condition means that the Hessian of $f_{u}$ on $\mathscr{N}^{\lambda}$ at $\left(x^{0}, \zeta^{2}\left(x^{0}\right)\right)$ is positive definite. In terms of a chart $\left(\theta_{1}, \cdots, \theta_{k}\right)$ of $\mathscr{N}^{2}$ with center $\left(x^{0}, \zeta^{2}\left(x^{0}\right)\right)$, this means that the $k \times k$ matrix $\left(\partial^{2} f_{u} / \partial \theta_{\gamma} \partial \theta_{\gamma^{\prime}}\right)(0)$ is positive definite. If $\left(x^{0}, \zeta^{2}\left(x^{0}\right)\right)$ is nondegenerate for a choice of a pair of $\mathscr{N}^{2}$ and a local trivialization of $E$, it is also so for any other such choice. We can check this by writing down how $f_{u}$ and its Hessian change when we make a different choice. Note on this connection that $a\left(x^{0}, \zeta^{2}\left(x^{0}\right)\right)$ $\cdot \rho_{1}^{\lambda}\left(x^{0}, \zeta^{\lambda}\left(x^{0}\right)\right)=0$.

Because of (9) and (10), $\left\{\left(w,\left(\zeta^{\lambda}\left(x^{0}\right)+\chi\right) /\left(1+|\chi|^{2}\right)^{\frac{1}{2}}\right) ; w \in N^{\lambda}\right.$ and $\left.\chi \perp \zeta^{\lambda}\left(x^{0}\right)\right\}$ forms a submanifold $\zeta^{2}$ as above. Hence by (11) and (15), the nondegeneracy condition means that $F^{\lambda}\left(x^{0} ; w, \chi\right) \mid W^{\lambda}\left(x^{0}, \zeta^{\lambda}\left(x^{0}\right)\right)$ is injective for all $w \in T_{x^{0}} N^{\lambda}$ and $\chi \perp \zeta^{2}\left(x^{0}\right)$. Thus we have

Proposition 2.1. Assume that the characteristics of $A$ are smooth and the projection $C^{\lambda} \rightarrow{ }^{\prime} C^{\lambda}$ is bijective, and further that $\left(x^{0}, \zeta^{\lambda}\left(x^{0}\right)\right)$ is a nondegenerate characteristic. Then $F^{\lambda}\left(x^{0} ; w, \chi\right)$, restricted to $W\left(x^{0}, \zeta^{2}\left(x^{0}\right)\right)$, is injective for sufficiently small $w \in N^{\lambda}$ and any $\chi \perp \zeta^{\lambda}\left(x^{0}\right)$ provided $(w, \chi) \neq 0$.

Lemma 2.8. Under the assumptions in Proposition 2.1, for any $\varepsilon>0$ we

\footnotetext{
Received August 11, 1971. Partially supported by the National Science Foundation under Grant No. GP-8988; continuation of Part I, J. Differential Geometry 6 (1972 421-436.
} 
can find $\delta_{\varepsilon}>0$ satisfying the following condition: For any $\delta_{\varepsilon}>\delta>0$ there is $C_{\varepsilon, r}$ such that

$$
\mathscr{R}\left\langle K_{\delta 2}^{\lambda}(x, D) u, u\right\rangle+C_{\varepsilon, \delta}\|Q(u)\|^{2} \geq-\varepsilon\|u\|_{\frac{1}{2}}^{2}
$$

for all $u \in C_{0}^{\infty}\left(U, U \times L_{0}\right)$, provided we choose $U$ sufficiently small and $\varphi^{\lambda}$ with its support sufficiently close to $C^{\lambda}$.

Proof. By Proposition 2.1, $F^{\lambda}(y ; w, \chi)$ on $W^{\lambda}\left(y, \zeta^{\lambda}(y)\right)$ is injective when $(w, \chi) \neq 0$ and $\chi \perp \zeta^{\lambda}(y)$ provided $|y|$ and $|w|$ are sufficiently small. On the other hand, $H_{2}^{\lambda}(x, \xi)$ is of order at least 3 in $(w, \chi(x, \xi))$. Hence for any $\delta>0$ there is $\delta(\varepsilon)>0$ such that

$$
\left\langle\delta F^{\lambda}\left(y ; w\left\langle\zeta^{\lambda}(y), \xi\right\rangle, \chi\right)^{*} F^{\lambda}\left(y ; w\left\langle\zeta^{\lambda}(y), \xi\right\rangle, \chi\right)+H_{2}^{\lambda}(x, \xi) u, u\right\rangle>0,
$$

where $\chi=\chi(y, \xi)$ for all nonzero $u \in W^{\lambda}\left(y, \zeta^{\lambda}(y)\right)$ provided $|y|,|w|$ and $|\xi|^{-1} \chi(y, \xi)$ are less than $\delta(\varepsilon)$. Since $W^{\lambda}\left(y, \zeta^{\lambda}(y)\right)$ is the image of $\rho_{1}^{\lambda}(y, \xi)$, we see by (22) that

$$
\left\langle K_{\delta 2}^{\lambda}(x, \xi) u, u\right\rangle>0
$$

for all $u \in L_{0}$, provided we choose $U$ sufficiently small and $\varphi^{2}$ with its support sufficiently close to $C^{\lambda}$. Hence by Theorem 1.4 ,

$$
\left\langle K_{\delta 2}^{\lambda}(x, D) u, u\right\rangle \geq-\langle L(x, D) u, u\rangle,
$$

where

$$
\begin{aligned}
L(x, \xi)= & \sum \frac{1}{2}\left(1+|\xi|^{2}\right)^{\frac{1}{2}} a_{j k} \partial^{2} K_{\delta 2}^{\lambda}(x, \xi) / \partial \xi_{j} \partial \xi_{k} \\
& +\left(1+|\xi|^{2}\right)^{-\frac{1}{2}} b_{j k} \partial^{2} K_{\delta 2}^{\lambda}(x, \xi) / \partial x_{j} \partial x_{k}+\text { terms of lower orders, }
\end{aligned}
$$

and $a_{j k}, b_{j k}$ are given in (12) of $\S 1$. By (22) and (23) we see easily that each component of the matrices $\partial^{2} K_{\delta 2}^{\lambda}(x, \xi) / \partial \xi_{j} \partial \xi_{k}$ and $\partial^{2} K_{\delta 2}^{2}(x, \xi) / \partial x_{j} \partial x_{k}$ can be written as $\varphi^{\lambda}(x, \xi)^{2}(\delta t(x, \xi)+h(x, \xi))+s(x, \xi)$, where $t$ and $h$ are independent of the choice of $\varphi^{\lambda}$ and Supp $s(x, \xi)$ does not touch the characteristics. Moreover, $h(x, \xi)$ is of order at least 1 in $w_{1}, \cdots, w_{n-1}, \chi_{1}(x, \xi), \cdots, \chi_{n-1}(x, \xi)$. Thus we can suppose that $\left|\varphi^{\lambda}(x, \xi)^{2}(\delta t(x, \xi)+h(x, \xi))\right| /|\xi|$ is as small as we wish, when $\delta$ and $U$ are sufficiently small and $\operatorname{Supp} \varphi^{\lambda}(x, \xi)$ is sufficiently close to $C^{\lambda}$. From this together with Lemma 2.3, we therefore see that

$$
|\mathscr{R}\langle L(x, D) u, u\rangle| \leq \varepsilon\left(\|u\|_{\frac{1}{2}}\right)^{2}+C Q(u) .
$$

Hence $\mathscr{R}\left\langle K_{\delta 2}^{\lambda}(x, D) u, u\right\rangle+C Q(u) \geq-\varepsilon\left(\|u\|_{\frac{1}{2}}\right)^{2}$. q.e.d.

By Lemmas 2.7 and 2.8, we have

Lemma 2.9. Under the assumptions in Lemma 2.8, for any $\varepsilon>0$ we can find $\delta_{\varepsilon}$ such that for any $\delta_{\varepsilon}>\delta>0$ we have

$$
C_{\varepsilon, \delta} Q(u)+\varepsilon\|u\|_{\frac{1}{2}}^{2} \geq(1-\delta)\left\|F^{\lambda}\left\{\varphi^{\lambda}(x, D) \rho_{1}^{\lambda}(x, D)\right\} u\right\|^{2} \quad\left(u \in C_{0}^{\infty}\left(U, U \times L_{0}\right)\right)
$$


provided $C_{\varepsilon, \delta}$ is sufficiently large, $U$ is sufficiently small, and Supp $\varphi^{\lambda}$ is sufficiently close to $C^{\lambda}$.

We further reduce the problem to a case of a differential operator with constant coefficient in $w\left\langle\zeta^{2}(y), D\right\rangle$ and $\chi(x, D)$.

Lemma 2.10. Under the assumptions in Proposition 2.1, for any $\varepsilon>0$ we can find $\delta_{\varepsilon}$ such that for any $0<\delta<\delta_{\varepsilon}$ we have the following: If $U$ is suffciently small and Supp $\varphi^{\lambda}$ is sufficiently close to $C^{\lambda}$, then

$$
\begin{aligned}
\left\|F^{\lambda}\left(y ; w\left\langle\zeta^{\lambda}(y), D\right\rangle, \chi(y, D)\right)\left\{\varphi^{\lambda}(x, D) \rho_{1}^{\lambda}(x, D)\right\} u\right\|^{2} \\
-(1-\delta)\left\|F^{\lambda}\left(0 ; w\left\langle\zeta^{\lambda}(y), D\right\rangle, \chi(y, D)\right)\left\{\varphi^{\lambda}(x, D) \rho_{1}^{\lambda}(x, D)\right\} u\right\|^{2} \\
\quad \geq \varepsilon\|u\|_{\frac{1}{2}}^{2}-C\|u\|^{2}-\|R(x, D) u\|^{2}
\end{aligned}
$$

for all $u \in C_{0}^{\infty}\left(U, U \times L_{0}\right)$, where $R(x, \xi)$ depends on $\varphi^{\lambda}$, is of order 1 , and Supp $R$ is outside of the characteristics.

Proof. By Proposition 2.1 there is a constant $c>0$ such that

$$
\left|F^{\lambda}(y ; w, \chi) u\right|^{2} \geq c\left(|w|^{2}+|\chi|^{2}\right)|u|^{2},
$$

provided $y, w$ are sufficiently small, $\chi \perp \zeta^{2}(y)$, and $u /|u|$ is in a sufficiently small neighborhood of $W\left(x^{0}, \zeta^{\lambda}\left(x^{0}\right)\right)$. Since $F^{\lambda}(y ; w, \chi)$ is linear in $w$ and $\chi$, it follows

$$
\begin{aligned}
& \left|\varphi^{\lambda}(x, \xi) F^{\lambda}\left(y ; w\left\langle\zeta^{\lambda}(y), \xi\right\rangle, \chi(y, \xi)\right) \rho_{1}^{\lambda}(x, \xi) u\right|^{2} \\
& \quad \geq c\left(|w|^{2}\left\langle\zeta^{\lambda}(y), \xi\right\rangle^{2}+|\chi(y, \xi)|^{2}\right) \varphi^{\lambda}(x, \xi)^{2}\left|\rho_{1}^{\lambda}(x, \xi) u\right|^{2}
\end{aligned}
$$

for all $u \in L_{0}$ and $x \in U$, provided $U$ is sufficiently small and $\operatorname{Supp} \varphi^{\lambda}$ is sufficiently close to $C^{\lambda}$. For any $\varepsilon_{1}>0$ we may also assume that $U$ is so small that

$$
\left.|| F^{\lambda}(y ; w, \chi) u\right|^{2}-\left.\left|F^{\lambda}(0 ; w, \chi) u\right|^{2}\left|\leq \varepsilon_{1}\left(|w|^{2}+|\chi|^{2}\right)\right| u\right|^{2}
$$

Set

$$
\begin{aligned}
G(x, \xi) & =F^{\lambda}\left(y ; w\left\langle\zeta^{\lambda}(y), \xi\right\rangle, \chi(y, \xi)\right), \\
G_{0}(x, \xi) & =F^{\lambda}\left(0 ; w\left\langle\zeta^{\lambda}(y), \xi\right\rangle, \chi(y, \xi)\right) .
\end{aligned}
$$

Then

$$
\begin{aligned}
&\left|G(x, \xi) \varphi^{\lambda}(x, \xi) \rho_{1}^{\lambda}(x, \xi) u\right|^{2}-(1-\delta)\left|G_{0}(x, \xi) \varphi^{\lambda}(x, \xi) \rho_{1}^{\lambda}(x, \xi) u\right|^{2} \\
&= \delta\left|G(x, \xi) \varphi^{\lambda}(x, \xi) \rho_{1}^{\lambda}(x, \xi) u\right|^{2} \\
&+(1-\delta)\left(\left|G(x, \xi) \varphi^{\lambda}(x, \xi) \rho_{1}^{\lambda}(x, \xi) u\right|^{2}-\left|G_{0}(x, \xi) \varphi^{\lambda}(x, \xi) \rho_{1}^{\lambda}(x, \xi) u\right|^{2}\right) \\
& \geq\left(\delta c-(1-\delta) \varepsilon_{1}\right) \varphi^{\lambda}(x, \xi)^{2}\left(\left|w\left\langle\zeta^{\lambda}(y), \xi\right\rangle\right|^{2}+|\chi(y, \xi)|^{2}\right)\left|\rho_{1}^{\lambda}(x, \xi) u\right|^{2}
\end{aligned}
$$

For a given $\delta>0$ we choose $\varepsilon_{1}$ so small that $\delta c-(1-\delta) \varepsilon_{1}>0$. Then $\left\langle\varphi^{\lambda}(x, \xi)^{2} J(x, \xi) u, u\right\rangle \geq 0$, where 


$$
J(x, \xi)=\rho_{1}^{\lambda}(x, \xi)\left(G(x, \xi) * G(x, \xi)-(1-\delta) G_{0}(x, \xi) * G_{0}(x, \xi)\right) \rho_{1}^{2}(x, \xi) .
$$

Hence by Theorem 1.4,

$$
\left\langle\left\{\varphi^{\lambda}(x, D)^{2} J(x, D)\right\} u, u\right\rangle \geq-\mathscr{R}\langle L(x, D) u, u\rangle,
$$

where

$$
\begin{gathered}
L(x, \xi)=\varphi^{2}(x, \xi)^{2} \sum_{j, k}\left(\frac{1}{2}(1+|\xi|)^{\frac{1}{2}} a_{j k} \partial^{2} J(x, \xi) / \partial \xi_{j} \partial \xi_{k}\right. \\
\left.+\frac{1}{2}(1+|\xi|)^{-\frac{1}{2}} b_{j k} \partial^{2} J(x, \xi) / \partial x_{j} \partial x_{k}\right)+R_{1}(x, \xi) \\
+ \text { terms of lower orders, }
\end{gathered}
$$

where $R_{1}(x, \xi)$ is a sum of terms containing derivatives of $\varphi^{\lambda}(x, \xi)$ and hence its support does not touch the characteristics. By (12) of $\S 1$, we may choose $g(x)$ in Theorem 1.4 in such a way that the absolute value of each component of the matrix $\sum \frac{1}{2}\left(1+|\xi|^{2}\right)^{-\frac{1}{2}} b_{j k} \partial^{2} J(x, \xi) / \partial x_{j} \partial x_{k}$ is less than $\varepsilon^{\prime}|\xi| . \partial^{2} J(x, \xi) / \partial \xi_{j} \partial \xi_{k}$ is a sum of terms which contain as a factor $G(x, \xi) * G(x, \xi)-(1-\delta) G_{0}(x, \xi) *$ - $G_{0}(x, \xi)$ or its partial derivatives in $\xi$. Since these partial derivatives can enter only through partial derivatives of $\left\langle\zeta^{\lambda}(y), \xi\right\rangle$ or of $\chi(y, \xi)$, each term contains a factor of the form $(a(y)-(1-\delta) a(0)) b(x, \xi)$, so that if we choose $\delta$ and $U$ sufficiently small, its absolute value can be made to be less than $\varepsilon^{\prime}|\xi|$. Thus for any $\varepsilon>0$ we find for a sufficiently small choice of $\varepsilon^{\prime}, \delta$, and $U$ that

$$
\left|\left\langle\left\{\varphi^{\lambda}(x, D)^{2} L(x, D)\right\} u, u\right\rangle\right| \leq \varepsilon\|u\|_{\frac{1}{2}}^{2}+\left|\left\langle R_{1}(x, D) u, u\right\rangle\right|
$$

for all $u \in C_{0}^{\infty}\left(U, U \times L_{0}\right)$. Therefore

$$
\begin{aligned}
\left\|F^{\lambda}\left(y ; w\left\langle\zeta^{\lambda}(y), D\right\rangle, \chi(y, D)\right)\left\{\varphi^{\lambda}(x, D) \rho_{1}^{\lambda}(x, D)\right\} u\right\|^{2} \\
\quad-(1-\delta)\left\|F^{\lambda}\left(0 ; w\left\langle\zeta^{\lambda}(y), D\right\rangle, \chi(y, D)\right)\left\{\varphi^{\lambda}(x, D) \rho_{1}^{\lambda}(x, D)\right\} u\right\|^{2} \\
=\left\langle\left(\left\{\varphi^{\lambda}(x, D)^{2} J(x, D)\right\}+B(x, D)\right) u, u\right\rangle \\
\geq\langle B(x, D) u, u\rangle-\varepsilon\|u\|_{\frac{1}{2}}^{2}-\left|\left\langle R_{1}(x, D) u, u\right\rangle\right|,
\end{aligned}
$$

where $B(x, \xi)$ is of order 1 and can be calculated by means of the formula for the symbols of compositions and adjoints of pseudo-differential operators. Hence it remains to show that we may assume

$$
|\mathscr{R}\langle B(x, D) u, u\rangle| \leq \varepsilon\|u\|_{\frac{1}{2}}^{2}+\left|\left\langle R_{2}(x, D) u, u\right\rangle\right|+\left|\left\langle T_{0} u, u\right\rangle\right|,
$$

where $R_{2}(x, \xi)$ is of order $\leq 1$ and does not touch the characteristics. We see easily that each term of the 1 st order part of $B(x, \xi)$ contains either $\varphi^{\lambda}(x, \xi)^{2}$ $\cdot w\left\langle\zeta^{2}(y), \xi\right\rangle, \varphi^{2}(x, \xi)^{2} \chi(y, \xi)$, a factor of the form $(a(y)-(1-\delta) a(0))$, or a derivative of $\varphi^{\lambda}(x, \xi)$. The sum of the terms of the last type is $R_{2}(x, \xi)$. We may assume that the absolute values of other terms are less than $\varepsilon^{\prime}|\xi|$. Hence for a sufficiently small choice of $\varepsilon^{\prime}$ we have the formula (26). q.e.d. 
By Lemmas 2.9 and 2.3 we have the following:

Proposition 2.2. Assume that the characteristics of $A$ are smooth, $C^{\lambda} \rightarrow^{\prime} C^{\lambda}$ is bijective, and the characteristics are nondegenerate at $\left(x^{0}, \zeta^{2}\left(x^{0}\right)\right)$. Then, for any $\varepsilon>0$ by a choice of a sufficiently small neighborhood $U$ of $x^{0}$ and $\varphi^{\lambda}(x, \xi)$ with its support sufficiently close to $C^{2}$,

$$
C_{\varepsilon}\|Q(u)\|^{2}+\varepsilon\|u\|_{\frac{1}{2}}^{2} \geq\left\|F^{\lambda}\left(x^{0} ; w\left\langle\zeta^{\lambda}(y), D\right\rangle, \chi(y, D)\right)\left\{\varphi^{\lambda}(x, D) \rho_{1}^{\lambda}(x, D)\right\} u\right\|^{2}
$$

for all $u \in C_{0}^{\infty}\left(U, U \times L_{0}\right)$ provided $C_{\varepsilon}$ is sufficiently large.

Expanding $a(x, \xi) \rho_{1}^{\lambda}(x, \xi) \rho_{2}^{\lambda}(x, \xi)=0$ in Taylor series in $(w, \chi)$ at $\left(y,\left\langle\xi, \zeta^{\lambda}(y)\right\rangle \zeta^{\lambda}(y)\right)$ and noting that $a\left(y, \zeta^{\lambda}(y)\right) \rho_{1}^{\lambda}\left(y, \zeta^{\lambda}(y)\right)=0$ we find that

$$
F^{\lambda}(y ; w, \chi) \rho_{2}^{\lambda}\left(y, \zeta^{\lambda}(y)\right)=0 .
$$

In particular,

$$
F^{\lambda}\left(x^{0} ; w, \chi\right) \rho_{2}^{\lambda}\left(x^{0}, \zeta^{\lambda}\left(x^{0}\right)\right)=0,
$$

and we may consider that

$$
F^{\lambda}\left(x^{0} ; w, \chi\right) \in \operatorname{Hom}\left(W^{\lambda}\left(x^{0}, \zeta^{\lambda}\left(x^{0}\right)\right), E_{0}\right)
$$

Definition 2.4. Assume that the characteristics of $A$ are smooth. We say that the characteristics are of fiber dimension 0 if and only if, for each point $x^{0}$ of $M$ and for each component $C^{\lambda}$ of the characteristics passing over $x^{0}, \pi: C^{\lambda}$ $\rightarrow{ }^{\prime} C^{\lambda}$ is bijective.

Proposition 2.3. Assume that the characteristics of $A$ are smooth, of fiber dimension 0 , and nondegenerate, and further that there is $r \geq 0$ such that for each $\lambda$ we have, for all sufficiently small $\theta>0$,

$$
\begin{aligned}
&\left\|F^{\lambda}\left(x^{0} ; w\left\langle\zeta^{\lambda}(y), D\right\rangle, \chi(y, D)\right) v\right\|^{2}+C_{\theta}\|v\|^{2} \\
& \geq-C \theta^{r+\frac{1}{2}}\|v\|_{\frac{1}{2}}^{2}+c_{0} \theta^{r}\left\langle\left\langle\zeta^{\lambda}\left(x^{0}\right), D\right\rangle v, v\right\rangle+\left\langle L_{\theta}\left\langle\zeta^{\lambda}\left(x^{0}\right), D\right\rangle v, v\right\rangle \\
&+\mathscr{R}\left\langle\left(T_{1}^{\lambda \theta}(x, D)+R^{\lambda \theta}\left(x ; w\left\langle\zeta^{\lambda}(y), D\right\rangle, \chi(y, D)\right)\right) v, v\right\rangle
\end{aligned}
$$

for all $v \in C_{0}^{\infty}\left(U, W^{\lambda}\left(x^{0}, \zeta^{\lambda}\left(x^{0}\right)\right)\right)$, where $L_{\theta}$ is an endomorphism of $W^{\lambda}\left(x^{0}, \zeta^{2}\left(x^{0}\right)\right)$ such that $\left\langle L_{\theta} v, v\right\rangle \geq 0$ for all $v, T_{1}^{\lambda \theta}$ is of order $1, T_{1}^{\lambda \theta}\left(x^{0}, \xi\right)=0$ for all $\xi$, and $R^{\theta}(x ; w, \chi)$ is linear in $(w, \chi)$. Then, for a sufficiently small neighborhood $U$ of $x^{0}, Q(u) \geq c\|u\|_{\frac{1}{2}}^{2}$ for all $u \in C_{0}^{\infty}\left(U, U \times L_{0}\right)$.

Proof. For simplicity we set $\rho^{\lambda}=\rho_{1}^{\lambda}\left(x^{0}, \zeta^{\lambda}\left(x^{0}\right)\right)$. Clearly

$$
\left\|\rho^{\lambda}\left\{\varphi^{\lambda}(x, D) \rho_{1}^{\lambda}(x, D)\right\} u\right\|_{\frac{1}{2}} \leq C\|u\|_{\frac{1}{2}}+C_{1}\|u\|,
$$

where $C_{1}$ may depend on $\varphi^{\lambda}$. Put 


$$
\begin{gathered}
K(x, \xi)=|\xi| \varphi(x, \xi)^{2}+\sum_{\lambda} \varphi^{\lambda}(x, \xi)^{2}\left(|\xi| \rho_{2}^{\lambda}(x, \xi)\right. \\
\left.+\rho_{1}^{\lambda}(x, \xi)\left\langle\zeta^{\lambda}\left(x^{0}\right), \xi\right\rangle\right) \rho^{\lambda} \rho_{1}^{\lambda}(x, \xi) \\
+\frac{1}{|\xi|} \rho_{1}^{\lambda}(x, \xi) F^{\lambda}\left(x^{0} ; w\left\langle\zeta^{\lambda}(y), \xi\right\rangle, \chi(y, \xi)\right)^{*} F^{\lambda}\left(x^{0} ;\right. \\
\left.w\left\langle\zeta^{\lambda}(y), \xi\right\rangle, \chi(y, \xi)\right) \rho_{1}^{\lambda}(x, \xi) .
\end{gathered}
$$

Since the characteristics are nondegenerate,

$$
\left\langle\left(K(x, \xi)-c_{1}|\xi|\right) u, u\right\rangle \geq 0
$$

for a constant $c_{1}$. Therefore, since $K(x, \xi)$ is of order 1 , Theorem 1.4 implies that

$$
\langle K(x, D) u, u\rangle \geq c_{1}\|u\|_{\frac{1}{2}}^{2}-C\|u\|^{2} .
$$

On the other hand, by Lemmas 2.3 and 2.6 together with Proposition 2.2,

$$
\begin{aligned}
& \langle K(x, D) u, u\rangle \leq \varepsilon\|u\|_{\frac{1}{2}}+C_{\varepsilon} Q(u) \\
& \quad+\sum_{\lambda}\left\langle\left\langle\zeta^{\lambda}\left(x^{0}\right), D\right\rangle \rho^{\lambda} \varphi^{\lambda}(x, D) \rho_{1}^{\lambda}(x, D) u, \rho^{\lambda} \varphi^{\lambda}(x, D) \rho_{1}^{\lambda}(x, D) u\right\rangle .
\end{aligned}
$$

Thus, by our assumption,

$$
\begin{aligned}
\sum_{\lambda}\left\|F^{\lambda}\left(x^{0} ; w\left\langle\zeta^{\lambda}(y), D\right\rangle, \chi(y, D)\right) \varphi^{\lambda}(x, D) \rho_{1}^{\lambda}(x, D) u\right\|^{2} \\
\left.=\sum_{\lambda}\left\|F^{\lambda}\left(x^{0} ; w\left\langle\zeta^{\lambda}(y), D\right\rangle, \chi(y, D)\right) \rho^{\lambda} \varphi^{\lambda}(x, D) \rho_{1}^{\lambda}(x, D) u\right\|^{2}, \quad \text { (by }(27)\right) \\
\geq \sum_{\lambda}\left(-C \theta^{r+\frac{1}{2}}\left\|\rho^{\lambda} \varphi^{\lambda}(x, D) \rho_{1}^{\lambda}(x, D) u\right\|_{\frac{1}{2}}^{2}\right. \\
\quad+c_{0} \theta^{r}\left\langle\left\langle\zeta^{\lambda}\left(x^{0}\right), D\right\rangle \rho^{\lambda} \varphi^{\lambda}(x, D) \rho_{1}^{\lambda}(x, D) u, \rho^{\lambda} \varphi^{\lambda}(x, D) \rho_{1}^{\lambda}(x, D) u\right\rangle \\
\quad+\mathscr{R}\left\langle\left( T_{1}^{\lambda \theta}(x, D)+R^{\lambda \theta}\left(x ; w\left\langle\zeta^{\lambda}(y), D\right\rangle,\right.\right.\right. \\
\left.\left.\quad \chi(y, D)) \rho^{\lambda} \varphi^{\lambda}(x, D) \rho_{1}^{\lambda}(x, D) u, \rho^{\lambda} \varphi^{\lambda}(x, D) \rho_{1}^{\lambda}(x, D) u\right\rangle\right)+\gamma \\
\geq-\theta^{r+\frac{1}{2}} c^{\prime}\|u\|_{\frac{1}{2}}^{2}+c_{0} \theta^{r}\left(\langle K(x, D) u, u\rangle-\varepsilon\|u\|_{\frac{1}{2}}-C_{s}^{\prime} Q(u)\right) \\
\quad+\left\langle T_{1}^{\theta}(x, D) u, u\right\rangle+\gamma, \quad(\text { by }(29) \text { and }(32)),
\end{aligned}
$$

where $T_{1}^{\theta}(x, \xi)=\sum_{\lambda} \rho_{1}^{\lambda}(x, \xi)\left(T_{1}^{\lambda \theta}(x, \xi)+R^{\lambda \theta}(x ; \chi(x, \xi))\right) \varphi^{\lambda}(x, \xi)^{2} \rho^{\lambda} \rho_{1}^{\lambda}(x, \xi)$, and $\gamma=\left\langle L_{\theta}\left\langle\zeta^{\lambda}\left(x^{0}\right), D\right\rangle \rho^{\lambda}\left\{\varphi^{\lambda}(x, D) \rho_{1}^{\lambda}(x, D)\right\} u, \rho^{\lambda}\left\{\varphi^{\lambda}(x, D) \rho_{1}^{\lambda}(x, D)\right\} u\right\rangle$. By choosing Supp $\varphi^{\lambda}$ sufficiently close to $C^{\lambda}$ and $U$ small, we may assume that $\left\langle L_{\theta}\left\langle\zeta^{\lambda}\left(x^{0}\right), \xi\right\rangle \varphi^{\lambda}(x, \xi)^{2} \rho^{\lambda} \rho_{1}^{\lambda}(x, \xi) u, \rho^{\lambda} \rho_{1}^{\lambda}(x, \xi) u\right\rangle \geq 0$, so that $\gamma \geq-C_{\theta}\|u\|^{2}$. Hence by (31),

$$
\begin{gathered}
\sum_{\lambda}\left\|F^{\lambda}\left(x^{0} ; w\left\langle\zeta^{\lambda}(y), D\right\rangle, \chi(y, D)\right)\left\{\varphi^{\lambda}(x, D) \rho_{1}^{\lambda}(x, D)\right\} u\right\|^{2}+C_{\theta, s}^{\prime \prime} Q(u) \\
\geq c_{0} \theta^{r}\left(c_{1}-\left(\varepsilon+\theta^{\frac{1}{2}} c^{\prime}\right)\right)\|u\|_{\frac{1}{2}}^{2}+\left\langle T_{1}^{\theta}(x, D) u, u\right\rangle
\end{gathered}
$$


Choose $\theta_{1}$ and $\varepsilon$ so small that $c_{1}-\left(\varepsilon+\theta_{1}^{\frac{1}{1}} c^{\prime}\right)>0$. Then, for constants $c>0$ and $C>0$ (setting $T_{1}(x, \xi)=T_{1}^{\theta_{1}}(x, \xi)$ ),

$$
\begin{aligned}
& \sum_{\lambda}\left\|F^{\lambda}\left(x^{0} ; w\left\langle\zeta^{\lambda}(y), D\right\rangle, \chi(y, D)\right)\left\{\varphi^{\lambda}(x, D) \rho_{1}^{\lambda}(x, D)\right\} u\right\|^{2}+C Q(u) \\
& \geq c\|u\|_{\frac{1}{2}}^{2}+\left\langle T_{1}(x, D) u, u\right\rangle
\end{aligned}
$$

for all $u \in C_{0}^{\infty}\left(U, U \times L_{0}\right)$, provided $U$ is sufficiently small and Supp $\varphi^{\lambda}$ is sufficiently close to $C^{\lambda}$. Now by applying Proposition 2.2 to the left hand side of (33) for a sufficiently small choice of $\varepsilon$, for constants $c>0$ and $C>0$ (where $c$ is independent of but $C$ is dependent on the choice of $\varphi^{\lambda}$ ) we find that

$$
C Q(u) \geq c\|u\|_{\frac{1}{2}}^{2}+\left\langle T_{1}(x, D) u, u\right\rangle .
$$

Since $T_{1}(x, \xi)=\sum_{\lambda}\left(T_{1}^{\lambda \theta_{1}}(x, \xi)+R^{\lambda \theta_{1}}(x ; \chi(x, \xi))\right) \varphi^{\lambda}(x, \xi)^{2} \rho^{\lambda} \rho_{1}^{\lambda}(x, \xi)$ and $T_{1}^{\lambda \theta}\left(x^{0}, \xi\right)$ $=0, T_{1}(x, \xi) /|\xi|$ can be made arbitrarily small by choosing $U$ sufficiently small and $\operatorname{Supp} \varphi^{\lambda}$ sufficiently close to $C^{\lambda}$. Hence for such choice of $U$ and $\varphi^{\lambda}$, $\frac{c}{2}\|u\|_{\frac{1}{2}}^{2} \leq C Q(u)$.

Definition 2.5. $F^{\lambda}\left(x^{0} ; w\left\langle\zeta^{\lambda}(y), D\right\rangle, \chi(y, D)\right)$ will be called the localized operator of $A$ at $x^{0}$ for the characteristics $C^{\lambda}$. For $\eta=\left(\eta_{1}, \cdots, \eta_{2 n-2}\right) \in \boldsymbol{R}^{2(n-1)}$, $g^{\lambda}(\eta)=F^{\lambda}\left(x^{0} ; \eta_{1}, \cdots, \eta_{2 n-2}\right)$ will be called the indirect symbol of the localized operator. (We recall $w$ and $y$ are considered as functions of $x$.)

In order to make the writing easy, we fix $\lambda$ once for all and set for $j=1, \cdots, n-1$

$$
\begin{gathered}
X_{j}(x, \xi)=w_{j}(x)\left\langle\zeta^{\lambda}(y(x)), \xi\right\rangle, \quad X_{j}=X_{j}(x, D), \\
X_{n-1+j}(x, \xi)=\chi_{j}(x, \xi), \quad X_{n-1+j}=X_{n-1+j}(x, D), \\
f^{\lambda j}\left(x^{0}\right)=f^{j}, \quad g^{\lambda j}\left(x^{0}\right)=f^{n-1+j} .
\end{gathered}
$$

Thus we can write

$$
F^{2}=F^{\lambda}\left(x^{0} ; w\left\langle\zeta^{2}(y), D\right\rangle, \chi(y, D)\right)=\sum_{s=1}^{2 n-2} f^{s} X_{s},
$$

By direct calculation we find that for $s, t=1, \ldots, 2 n-2$

$$
X_{s}^{*} X_{t}-X_{t}^{*} X_{s}=\frac{1}{i} c_{s t}(x)\left\langle\zeta^{\lambda}(y), D\right\rangle+\sum_{i=1}^{2 n-1} b_{s t}^{r}(x) X_{r}
$$

where $c_{s t}(x)$ is a real valued function, skew-symmetric in $s, t$, given by, for $j, k=1, \cdots, n-1$, 


$$
\begin{aligned}
& c_{j k}(x)=0, \\
& c_{n-1+j k}(x) \equiv i X_{n-1+j} w_{k}(x) \quad(\bmod w), \\
& c_{n-1+j n-1+k}(x) \equiv i\left(X_{n-1+j} \zeta_{k}^{\lambda}(x)-X_{n+1+k} \zeta_{j}^{2}(x)\right), \quad\left(\bmod \zeta^{2}\right) .
\end{aligned}
$$

Another way of writing down these functions are as follows:

$$
\begin{aligned}
d w_{j} & \equiv \sum_{k} c_{b+j k} \chi_{k}(y, d x) \quad\left(\bmod \left\langle\zeta^{\lambda}(y), d x\right\rangle\right), \\
d \zeta^{\lambda} & \equiv \sum_{j, k} \frac{1}{2} c_{n-1+k} n_{n-1+j} \chi_{j}(y, d x) \wedge \chi_{k}(y, d x), \quad\left(\bmod \left\langle\zeta^{\lambda}(y), d x\right\rangle\right),
\end{aligned}
$$

where $\zeta^{2}$ denotes the differential form $\left\langle\zeta^{\lambda}(y(x)), d x\right\rangle=\sum_{j} \zeta_{j}^{\lambda}(y(x)) d x_{j}$.

\section{Study of the characteristic parts}

In this section we fix vector spaces $W, V$, and a linear mapping

$$
g: R^{2 n-2} \ni \eta \rightarrow g(\eta) \in \operatorname{Hom}(W, V) .
$$

We write

$$
g(\eta)=\sum_{s=1}^{2 n-2} g^{s} \eta_{s}
$$

where $g^{s} \in \operatorname{Hom}(W, V)$. $U$ will be as in $\S 2, T_{j}$ will denote as in $\S 2$ the pseudodifferential operators of order $j$ which may change from formulas to formulas, and $X_{1}, \cdots, X_{2 n-2}$ will have the same meaning as in $\S 2$. For $u \in C_{0}^{\infty}(U, W)$ we set

$$
g(X) u=\sum g^{s} X_{s} u,
$$

which is in $C_{0}^{\infty}(U, V)$. We are interested in an estimate of the type described in Proposition 2.3. To this end we apply our results to the case where $\boldsymbol{g}(\eta)=$ $F^{\lambda}\left(x^{0} ; \eta\right), W=W^{\lambda}\left(x^{0}, \zeta^{\lambda}\left(x^{0}\right)\right)$ and $V=E_{0}$. Assume that we are given hermitian metrics on $W, V$, and set

$$
\Delta_{g}(x, \xi)=g(X(x, \xi))^{*} g(X(x, \xi)) .
$$

Lemma 3.1. For $u \in C_{0}^{\infty}(U, W)$,

$$
\begin{aligned}
\|g(X) u\|^{2}= & \mathscr{R}\left\langle\Delta_{g}(x, D) u, u\right\rangle \\
& +\sum \frac{1}{2} \mathscr{R}\left\langle g^{s^{*}} g^{t}\left(\frac{1}{i} c_{s t}(x)\left\langle\zeta^{2}(y), D\right\rangle+b_{s t}^{r}(x) X_{r}\right) u, u\right\rangle \\
& +\langle g(X) u, g(h(x)) u\rangle+\left\langle T_{0}(x, D) u, u\right\rangle,
\end{aligned}
$$

where $h_{s}(x)$ is a $C^{\infty}$ function, $c_{s t}(x)$ and $b_{s t}^{r}(x)$ are defined in $\S 2$ of (38). 
Proof. Clearly, $X_{s}^{*}=X_{s}+h_{s}(x)$, where $h_{s}(x)$ is $a C^{\infty}$ function. Thus

$$
\begin{aligned}
g(X)^{*} g(X) u= & \Delta_{g}(x, D) u+\sum_{s, t, j} g^{s^{*}} g^{t}\left(\partial X_{s}(x, \xi) / \partial \xi_{j}\right) Y_{t j}(x, \xi) u \\
& +g(h(x))^{*} \boldsymbol{g}(X) u
\end{aligned}
$$

where $Y_{t j}=(1 / i) \partial X_{t}(x, \xi) / \partial x_{j}$, and therefore

$$
\begin{aligned}
\|\boldsymbol{g}(X) u\|^{2}= & \mathscr{R}\left\langle\Delta_{\boldsymbol{g}}(x, D) u, u\right\rangle+\mathscr{R}\langle\boldsymbol{g}(X) u, \boldsymbol{g}(h(x)) u\rangle \\
& +\mathscr{R} \sum\left\langle g^{s^{*}} g^{t}\left(\partial X_{s}(x, \xi) / \partial \xi_{j}\right) Y_{t j}(x, D) u, u\right\rangle .
\end{aligned}
$$

(Note that $\partial X_{s} / \partial \xi_{j}$ is a function of $x$.) On the other hand, since $Y_{t j}(x, \xi)$ has purely imaginary coefficients, we have

$$
\begin{aligned}
\mathscr{R} \sum & \left\langle g^{s^{*}} g^{t}\left(\partial X_{s}(x, \xi) / \partial \xi_{j}\right) Y_{t j}(x, D) u, u\right\rangle \\
= & -\mathscr{R} \sum\left\langle g^{g^{*}} g^{t}\left(\partial X_{t}(x, \xi) / \partial \xi_{j}\right) Y_{s j}(x, D) u, u\right\rangle+\mathscr{R}\left\langle T_{0}(x, D) u, u\right\rangle \\
= & \frac{1}{2} \mathscr{R} \sum\left\langleg ^ { s ^ { * } } g ^ { t } \left\{\left(\partial X_{s}(x, \xi) / \partial \xi_{j}\right) Y_{t j}(x, D)\right.\right. \\
& \left.\left.\quad-\left(\partial X_{t}(x, \xi) / \partial \xi_{j}\right) Y_{s j}(x, D)\right\} u, u\right\rangle+\mathscr{R}\left\langle T_{0}(x, D) u, u\right\rangle \\
= & \frac{1}{2} \mathscr{R} \sum\left\langle g^{s^{*}} g^{t}\left(X_{s} X_{t}-X_{t} X_{s}\right) u, u\right\rangle+\mathscr{R}\left\langle T_{0}(x, D) u, u\right\rangle \\
= & \frac{1}{2} \mathscr{R} \sum\left\langle g^{s^{*}} g^{t}\left(X_{s}^{*} X_{t}-X_{t}^{*} X_{s}\right) u, u\right\rangle+\mathscr{R}\left\langle T_{0}(x, D) u, u\right\rangle \\
= & \frac{1}{2} \mathscr{R} \sum\left\langle g^{s^{*}} g^{t}\left(\frac{1}{i} c_{s t}(x)\left\langle\zeta^{2}(y), D\right\rangle+b_{s t}^{r}(x) X_{r}\right) u, u\right\rangle+\mathscr{R}\left\langle T_{0}(x, D) u, u\right\rangle,
\end{aligned}
$$

which together with (5) thus implies our formula. q.e.d.

Let $V^{\prime}$ be a vector space with a hermitian metric, and assume that we have, for all $\theta$ with sufficiently small absolute value, a linear map

$$
\boldsymbol{g}_{\theta}: \boldsymbol{R}^{2 n-2} \ni \eta \rightarrow \boldsymbol{g}_{\theta}(\eta) \in \operatorname{Hom}\left(W, V^{\prime}\right)
$$

which depends differentiably on $\theta$.

Lemma 3.2. Assume that for an integer $d \geq 1$ we have the following:

(i ) $\quad g^{s^{*}} g^{t}=g_{0}^{s^{*}} g_{0}^{t} \quad(s, t=1, \cdots, 2 n-2)$,

(ii) ${ }_{d} \quad \boldsymbol{g}(\eta)^{*} \boldsymbol{g}(\eta)-\boldsymbol{g}_{\theta}(\eta)^{*} \boldsymbol{g}_{\theta}(\eta)=\theta^{d+1} h_{\theta}(\eta)$,

where $h_{\theta}(\eta)$ depends differentiably on $\theta$, and

$$
\text { (iii) })_{d} \quad \sum\left\langle\frac{1}{i} c_{s t}\left(x^{0}\right)\left(g^{s^{*}} g^{t}-g_{\theta}^{s^{*}} g_{\theta}^{t}\right) u, u\right\rangle \geq c_{0} \theta^{d}|u|^{2}
$$

for all $u \in W$ and all sufficiently small $\theta>0$. Assume further that

(iv) $\left\langle\Delta_{g}(x, \xi) u, u\right\rangle \geq c_{1}\left(\sum_{s}\left|X_{s}(x, \xi) u\right|^{2}\right)$.

Then for sufficiently small $\theta>0$ 


$$
\begin{aligned}
\|\boldsymbol{g}(X) u\|^{2}+C_{\theta}\|u\|^{2} \geq & -C \theta^{d+\frac{1}{2}}\|u\|_{\frac{1}{2}}+c_{0} \theta^{d}\left\langle\left\langle\zeta^{2}\left(x^{0}\right), D\right\rangle u, u\right\rangle \\
& +\left\langle L_{\theta}\left\langle\zeta^{2}\left(x^{0}\right), D\right\rangle u, u\right\rangle+\left\langle\left(T_{1}^{\theta}(x, D)+R^{\theta}(x, X)\right) u, u\right\rangle
\end{aligned}
$$

for all $u \in C_{0}^{\infty}(U, W)$, where $T_{1}^{\theta}\left(x^{0}, \xi\right)=0$ for all $\xi, R^{\theta}(x, \eta)$ is linear in $\eta$, and $L_{\theta} \in \operatorname{Hom}(W, W)$ such that $\left\langle L_{\theta} u, u\right\rangle \geq 0$ for all $u$.

Proof. By Lemma 3.1,

$$
\begin{aligned}
\left\|\boldsymbol{g}_{\theta}(X) u\right\|^{2}= & \mathscr{R}\left\langle\Delta_{\boldsymbol{g}_{\theta}}(x, D) u, u\right\rangle+\mathscr{R} \sum \frac{1}{i}\left\langle\boldsymbol { g } _ { \theta } ^ { \boldsymbol { s } ^ { * } } \boldsymbol { g } _ { \theta } ^ { t } \left(\frac{1}{i} c_{s t}\left(x^{0}\right)\left\langle\zeta^{2}(y), D\right\rangle\right.\right. \\
& \left.\left.\left.+b_{s t}^{r} X_{r}\right)\right) u, u\right\rangle+\mathscr{R}\left\langle\boldsymbol{g}_{\theta}(X) u, \boldsymbol{h}_{\theta}(h(x)) u\right\rangle+\left\langle T_{0}^{\theta}(x, D) u, u\right\rangle
\end{aligned}
$$

Thus for $0<\delta<1$

$$
\begin{aligned}
\|\boldsymbol{g}(X) u\|^{2}= & \delta\|\boldsymbol{g}(X) u\|^{2}+(1-\delta)\left\|\boldsymbol{g}_{\theta}(X) u\right\|^{2} \\
& +(1-\delta)\left(\|\boldsymbol{g}(X) u\|^{2}-\left\|\boldsymbol{g}_{\theta}(X) u\right\|^{2}\right) \\
= & (1-\delta)\left\|\boldsymbol{g}_{\theta}(X) u\right\|^{2}+\mathscr{R}\left\langle\left(\delta \Delta_{\boldsymbol{g}}(x, D)+(1-\delta)\left(\Delta_{\boldsymbol{g}}(x, D)\right.\right.\right. \\
& \left.\left.\left.-\Delta_{g_{\theta}}(x, D)\right)\right) u, u\right\rangle \\
& +\mathscr{R} \sum\left\langle\frac{c_{s t}}{2 i}\left(\delta g^{s^{*}} g^{t}+(1-\delta)\left(g^{s^{*}} g^{t}-g_{\theta}^{s^{*}} g_{\theta}^{t}\right)\right)\left\langle\zeta^{\lambda}(y), D\right\rangle u, u\right\rangle \\
& +\mathscr{R}\left\langle\left(R^{\theta, \delta}(x, x)+T_{0}^{\theta, o}(x, D)\right) u, u\right\rangle .
\end{aligned}
$$

Hence

$$
\begin{aligned}
\|\boldsymbol{g}(X) u\|^{2} & +C_{\theta}\|u\|^{2} \\
\geq & \mathscr{R}\left\langle\left(\delta \Delta_{\boldsymbol{g}}(x, D)+(1-\delta)\left(\Delta_{\boldsymbol{g}}(x, D)-\Delta_{\boldsymbol{g}_{\theta}}(x, D)\right)\right) u, u\right\rangle \\
& +\mathscr{R} \sum \frac{1}{2 i}\left\langle c_{s t}\left(\delta g^{s^{*}} g^{t}+(1-\delta)\left(g^{g^{*}} g^{t}-g_{\theta}^{s^{*}} g_{\theta}^{t}\right)\right)\left\langle\zeta^{\lambda}(x), D\right\rangle u, u\right\rangle \\
& +\mathscr{R}\left\langle R^{\theta, \delta}(x, X) u, u\right\rangle .
\end{aligned}
$$

By (ii) $)_{d}$ and (iv),

$$
\begin{aligned}
& \left\langle\left(\delta \Delta_{g}(x, \xi)+(1-\delta)\left(\Delta_{g}(x, \xi)-\Delta_{g_{\theta}}(x, \xi)\right)\right) u, u\right\rangle \\
& \quad \geq \delta c_{1} \sum_{s}\left|X_{s}(x, \xi) u\right|^{2}-(1-\delta) \theta^{d+1}\left\langle h_{\theta}(X(x, \xi)) u, u\right\rangle .
\end{aligned}
$$

For $\theta>0$ and $\delta=\theta^{d+\frac{1}{2}}$, the right hand side of the above inequality becomes

$$
\theta^{d+\frac{1}{2}}\left(c_{1} \sum_{s}\left|X_{s}(x, \xi) u\right|^{2}-\left(1-|\theta|^{d+\frac{1}{2}}\right) \theta^{\frac{1}{2}}\left\langle h_{\theta}(X(s, \xi)) u, u\right\rangle\right) .
$$

Therefore for sufficiently small $\theta$, by Theorem 1.4 we have

$$
\left\langle\left(\delta \Delta_{g}(x, D)+(1-\delta)\left(\Delta_{g}(x, D)-\Delta_{g_{\theta}}(x, D)\right)\right) u, u\right\rangle \geq-C_{1} \theta^{d+\frac{1}{2}}\|u\|_{\frac{1}{2}},
$$


where $\delta=\theta^{d+\frac{1}{2}}$. By (iii) ${ }_{d}$ we have for $\delta=\theta^{d+\frac{1}{2}}$

$$
\begin{aligned}
\sum\langle & \left.\frac{1}{2 i} c_{s t}\left(x^{0}\right)\left(\delta g^{s^{*}} g^{t}+(1-\delta)\left(g^{s^{*}} g^{t}-g_{\theta}^{s^{*}} g_{\theta}^{t}\right)\right)\left\langle\zeta^{\lambda}\left(x^{0}\right), D\right\rangle u, u\right\rangle \\
& \geq C_{0} \theta^{d}\left\langle\left\langle\zeta^{\lambda}\left(x^{0}\right), D\right\rangle u, u\right\rangle-\theta^{d+\frac{1}{2}} c^{\prime}\|u\|_{\frac{1}{2}}^{2}+\left\langle L_{\theta}\left\langle\zeta^{\lambda}\left(x^{0}\right), D\right\rangle u, u\right\rangle,
\end{aligned}
$$

together with (6) and (7)

$$
\begin{aligned}
\|\boldsymbol{g}(X) u\|^{2}+C_{\theta}\|u\|^{2} \geq & c_{0} \theta^{d}\left\langle\left\langle\zeta^{\lambda}\left(x^{0}\right), D\right\rangle u, u\right\rangle-\theta^{d+\frac{1}{2}} c\|u\|_{\frac{1}{2}}^{2} \\
& +\mathscr{R}\left\langle R^{\theta, o}(x, X) u, u\right\rangle+\mathscr{R}\left\langle T_{1}^{\theta}(x, D) u, u\right\rangle \\
& +\left\langle L_{\theta}\left\langle\zeta^{2}\left(x^{0}\right), D\right\rangle u, u\right\rangle,
\end{aligned}
$$

where $T_{1}^{\theta}\left(x^{0}, \xi\right)=0$.

Theorem 3.1. Let $A$ be a pseudo-differential operator of order 1 mapping $C_{0}^{\infty}(M, L)$ into $C_{0}^{\infty}(M, E)$. Assume that the characteristics of $A$ are smooth, of fiber dimension 0 , and nondegenerate, and further that, for each $x^{0} \in M$ and each component of the characteristics $C^{\lambda}$ passing over $x^{0}$, the indirect symbol $\boldsymbol{g}(\eta)$ of the localized operator of $A$ at $x^{0}$ relative to $C^{\lambda}$ satisfies conditions (i), (ii) $_{d}$, and (iii) $)_{d}$ (for an integer $d \geq 1$ ) in Lemma 3.2. Then there is a constant $c>0$ such that

$$
\|A u\|^{2}+\|u\|^{2} \geq c\|u\|_{\frac{1}{2}}^{2}
$$

for all $u \in C^{\infty}(M, E)$.

Proof. If $\boldsymbol{g}(\eta)$ satisfies the conditions in Lemma 3.2 for $d \geq 1$, and $m$ is an integer $m \geq 1$, then $g(\eta)$ satisfies the conditions for $d m$, so that we may use the common $d$ for the indirect symbols relative to the components $C^{1}, \ldots, C^{\lambda}$, ... Hence our theorem is an immediate corollary of Proposition 2.3 and Lemma 3.2. q.e.d.

We further study the conditions in Lemma 3.2. For $g(\eta)=\sum g^{s} \eta_{s}$ they are conditions on $g^{s^{*}} g^{t} \in \operatorname{Hom}(W, W)$. Thus if we have another $\boldsymbol{h}(\eta) \in \operatorname{Hom}\left(W, E_{2}\right)$ such that $h^{s^{*}} h^{t}=g^{s^{*}} g^{t}$ for all $s, t=1, \cdots, 2 n-2$, and if $g(\eta)$ satisfies the conditions, then so does $\boldsymbol{h}(\eta) . g$ induces a linear mapping $g: W \otimes R^{2 n-2} \rightarrow E_{1}$, and vice versa, and $g$ and $g$ are related by

$$
g\left(u \otimes e^{s}\right)=g^{s} u \quad(u \in W),
$$

where $\left\{e^{s}\right\}$ is the standard base of $\boldsymbol{R}^{2 n-2}$. We impose the hermitian metric on $W \otimes \boldsymbol{R}^{2 n-2}$ induced by that of $W$. Let $h$ be the positive semidefinite hermitian square root of $g^{*} g$, and $\boldsymbol{h}(\eta) \in \operatorname{Hom}\left(W, W \otimes \boldsymbol{R}^{2 n-2}\right)$ be defined by $h$ as above. Then $\left\langle h^{s^{*}} h^{t} u, u^{\prime}\right\rangle=\left\langle h^{t} u, h^{s} u^{\prime}\right\rangle=\left\langle h\left(u \otimes e^{t}\right), h\left(u^{\prime} \otimes e^{s}\right)\right\rangle=\left\langle g\left(u \otimes e^{t}\right), g\left(u^{\prime} \otimes e^{s}\right)\right\rangle$ $=\left\langle g^{s^{*}} g^{t} u, u^{\prime}\right\rangle$, i.e., $h^{s^{*}} h^{t}=g^{s^{*}} g^{t}$. Thus we may replace $g$ by $h$. Moreover

$$
\operatorname{ker} g=\operatorname{ker} h \text {. }
$$


Hence we may assume without loss of generality that $V=W \otimes \boldsymbol{R}^{2 n-2}$, and that $g$ is a positive semidefinite hermitian metric. If $\boldsymbol{g}_{\theta}$ as in Lemma 3.2 exists, we may assume also that $\boldsymbol{g}_{\theta}(\eta) \in \operatorname{Hom}\left(W, W \otimes \boldsymbol{R}^{2 n-2}\right)$, or equivalently $g_{\theta} \in$ Hom $\left(W \otimes R^{2 n-2}, W \otimes R^{2 n-2}\right)$. Then the first condition says that $g^{*} g=g_{0}^{*} g_{0}$, i.e., $g=v g_{0}$ where $v$ is a unitary transformation of $W \otimes R^{2 n-2}$. Replacing $g_{\theta}$ by $v g_{\theta}$ we may assume that $g_{0}=g$.

We first study the conditions in Lemma 3.2 for the case $d=1$. Write

$$
g_{\theta} \equiv g+\theta r \quad\left(\bmod \theta^{2}\right)
$$

Then (ii) $)_{1}$ and (iii) $)_{1}$ are equivalent to

$$
\begin{aligned}
& \text { (ii) })_{1}^{\prime} \quad g^{s^{*}} r^{t}+r^{s^{*}} g^{t}+g^{t^{*}} r^{s}+r^{t^{*}} g^{s}=0, \\
& \text { (iii) })_{1}^{\prime} \quad\left\langle\frac{1}{i} c_{s t}^{0}\left(g^{s^{*}} r^{t}+r^{s^{*}} g^{t}\right) u, u\right\rangle<0
\end{aligned}
$$

for all nonzero $u$ in $W$, where $c_{s t}^{0}=c_{s t}\left(x^{0}\right)$. In order to write these conditions more concisely, we introduce an automorphism $\tau$ of $\operatorname{Hom}\left(W \otimes \boldsymbol{R}^{2 n-2}\right.$, $\left.W \otimes \boldsymbol{R}^{2 n-2}\right)$ defined by

$$
\left\langle r^{\tau}\left(u \otimes e^{s}\right), u^{\prime} \otimes e^{t}\right\rangle=\left\langle r\left(u \otimes e^{t}\right), u^{\prime} \otimes e^{s}\right\rangle
$$

for all $u, u^{\prime} \in W$ and $s, t=1, \cdots, 2 n-2$. Then (ii) ${ }_{1}^{\prime}$ is equivalent to

$$
\text { (ii) })_{1}^{\prime \prime} g^{*} r+r^{*} g+\left(g^{*} r\right)^{\tau}+\left(r^{*} g\right)^{\tau}=0,
$$

Let $J: \boldsymbol{R}^{2 n-2} \rightarrow \boldsymbol{R}^{2 n-2}$ be defined by

$$
J\left(e^{s}\right)=\sum_{t=1}^{2 n-2} c_{s t}^{0} e^{t}
$$

Then for $r, g \in \operatorname{Hom}\left(W \otimes \boldsymbol{R}^{2 n-2}, W \otimes \boldsymbol{R}^{2 n-2}\right)$,

$$
\begin{aligned}
\sum_{s, t}\left\langle c_{s t}^{0} r^{s^{*}} g^{t} u, u^{\prime}\right\rangle & =\sum\left\langle c_{s t}^{0} g\left(u \otimes e^{t}\right), r\left(u^{\prime} \otimes e^{s}\right)\right\rangle \\
& =\sum\left\langle g\left(u \otimes c_{s t}^{0} e^{t}\right), r\left(u^{\prime} \otimes e^{s}\right)\right\rangle \\
& =\sum\left\langle r^{*} g(I \otimes J)\left(u \otimes e^{s}\right), u^{\prime} \otimes e^{s}\right\rangle,
\end{aligned}
$$

where $I$ is the identity map of $W$. This suggests us to introduce a linear mapping $t r_{W}: \operatorname{Hom}\left(W \otimes \boldsymbol{R}^{2 n-2}, W \otimes \boldsymbol{R}^{2 n-2}\right) \rightarrow \operatorname{Hom}(W, W)$ defined by

$$
\left\langle\left(t r_{W} h\right) u, u^{\prime}\right\rangle=\sum_{s}\left\langle h\left(u \otimes e^{s}\right), u^{\prime} \otimes e^{s}\right\rangle \text {. }
$$

Then (iii) $)_{1}^{\prime}$ can be written as

$$
\text { (iii) })_{1}^{\prime \prime} \quad \operatorname{tr}_{W}\left(i\left(g^{*} r+r^{*} g\right)(I \otimes J)\right)>0 .
$$


Thus we have the following:

Lemma 3.3. Conditions (i), (ii) $)_{1}$, (iii) in Lemma 3.2 are satisfied if and only if we can find $r \in \operatorname{Hom}\left(W \otimes \boldsymbol{R}^{2 n-2}, W \otimes \boldsymbol{R}^{2 n-2}\right)$ such that

(ii) $)_{1}^{\prime \prime} g^{*} r+r^{*} g+\left(g^{*} r\right)^{\tau}+\left(r^{*} g\right)^{\tau}=0$,

(iii) $)_{1}^{\prime \prime} \quad t r_{W}\left(i\left(g^{*} r+r^{*} g\right)(I \otimes J)\right)>0$.

Let $V, V_{1}$ be vector spaces with hermitian metrics. Then we always consider the vector space $\operatorname{Hom}\left(V, V_{1}\right)$ with a hermitian metric defined by

$$
\langle r, g\rangle=\operatorname{Tr} g^{*} r \quad\left(g, r \in \operatorname{Hom}\left(V, V_{1}\right)\right) .
$$

The subspace over $\boldsymbol{R}$ of $\operatorname{Hom}(V, V)$ consisting of all self-adjoint transformations of $V$ will be denoted by $\operatorname{Her}(V, V)$, so that

$$
\operatorname{dim}_{R}(\operatorname{Her}(V, V))=\left(\operatorname{dim}_{C} V\right)^{2} .
$$

$\tau$ defined by the formula (9) is a hermitian unitary transformation of order 2 of $\operatorname{Hom}\left(W \otimes \boldsymbol{R}^{2 n-2}, W \otimes \boldsymbol{R}^{2 n-2}\right)$ and preserves $\operatorname{Her}\left(W \otimes \boldsymbol{R}^{2 n-2}, W \otimes \boldsymbol{R}^{2 n-2}\right)$. We set

$$
\begin{aligned}
S & =\left\{\alpha \in \operatorname{Her}\left(W \otimes \boldsymbol{R}^{2 n-2}, W \otimes \boldsymbol{R}^{2 n-2}\right) ; \alpha^{\tau}=\alpha\right\}, \\
S^{\Lambda} & =\left\{\beta \in \operatorname{Her}\left(W \oplus \boldsymbol{R}^{2 n-2}, W \otimes \boldsymbol{R}^{2 n-2}\right) ; \beta^{\tau}=-\beta\right\} .
\end{aligned}
$$

For a subspace $F$ of $V, \rho_{F}$ generally denotes the orthogonal projection of $V$ to $F$. We can now rewrite Lemma 3.3 as follows:

Proposition 3.1. Conditions (i), (ii) ${ }_{1}$, (iii) ${ }_{1}$ in Lemma 3.2 are satisfied if and only if there is $\beta \in S^{4}$ such that

1) $\rho_{K} \beta \rho_{K}=0 \quad$ where $K=\operatorname{ker} g$,

2) $\operatorname{tr}_{W}(i \beta(I \otimes J))>0$.

Proof. Assume that there is $r$ as in Lemma 3.3. Then $\beta=g^{*} r+r^{*} g$ is in $S^{\Lambda}$ and satisfies 1) and 2). Conversely, assume that $\beta$ satisfies 1) and 2). Since $\beta$ is hermitian, the condition 1$)$ implies that there is $r \in \operatorname{Hom}\left(W \otimes R^{2 n-2}\right.$, $W \otimes R^{2 n-2}$ ) such that $\beta=g^{*} r+r^{*} g$. Then this $r$ clearly satisfies (ii) ${ }_{1}^{\prime \prime}$ and (iii) $)_{1}^{\prime \prime}$. q.e.d.

In order to study these conditions further, we define a linear map (over $R$ ) $\theta: \operatorname{Hom}\left(W \otimes \boldsymbol{R}^{2 n-2}, W \otimes \boldsymbol{R}^{2 n-2}\right) \rightarrow \operatorname{Her}\left(W \otimes \boldsymbol{R}^{2 n-2}, W \otimes \boldsymbol{R}^{2 n-2}\right)$ by

$$
\theta(r)=g^{*} r+r^{*} g
$$

$r$ is in $\operatorname{Ker} \theta$ if and only if $i r^{*} g$ is hermitian. Since $i r^{*} g$ is zero on $\operatorname{Ker} g$, we thus have a linear map 


$$
\operatorname{Ker} \theta \rightarrow \operatorname{Her}(\operatorname{Im} g, \operatorname{Im} g) \text {, }
$$

where Im $g$ denotes the image of $g$ (being hermitian, it is the orthogonal complement of $\operatorname{Ker} g$ ). It is easy to check that this map is surjective and the kernel is isomorphic to Hom $\left(W \otimes \boldsymbol{R}^{2 n-2}, \operatorname{Ker} g\right)$. Thus

$$
\begin{aligned}
\operatorname{dim}_{R} \operatorname{Ker} \theta & =\left(\operatorname{dim}_{C} \operatorname{Im} g\right)^{2}+2 m(2 n-2) \operatorname{dim}_{C} \operatorname{Ker} g \\
& =((2 n-2) m)^{2}+\left(\operatorname{dim}_{C} \operatorname{Ker} g\right)^{2} \quad\left(m=\operatorname{dim}_{C} w\right) .
\end{aligned}
$$

When $V$ is a vector subspace of $W \otimes R^{2 n-2}, r \in \operatorname{Hom}(V, V)$ can be identified with an element in Hom $\left(W \otimes \boldsymbol{R}^{2 n-2}, W \otimes \boldsymbol{R}^{2 n-2}\right)$, which coincides with $r$ on $V$ and is zero on the orthogonal complement of $V$. Thus we always consider $\operatorname{Hom}(V, V)$ as a subspace of Hom $\left(W \otimes \boldsymbol{R}^{2 n-2}, W \otimes \boldsymbol{R}^{2 n-2}\right)$. We denote by $\pi_{S}$ the projection to $S$ of $S \oplus S^{\Lambda}=\operatorname{Her}\left(W \otimes R^{2 n-2}, W \otimes R^{2 n-2}\right)$. Clearly

$$
\pi_{S}(h)=\frac{1}{2}\left(h+h^{\tau}\right) \text {. }
$$

Lemma 3.4. $\left(\operatorname{Im}\left(\pi_{S} \circ \theta\right)\right)^{\perp} \cap S=\operatorname{Her}(\operatorname{Ker} g$, Ker $g) \cap S$, where $\perp$ is taken in $\operatorname{Her}\left(W \otimes \boldsymbol{R}^{2 n-2}, W \otimes \boldsymbol{R}^{2 n-2}\right)$.

Proof. $\alpha$ in $S$ is in $\left(\operatorname{Im}\left(\pi_{S} \circ \theta\right)\right)^{\perp} \cap S$ if and only if

$$
\left\langle r^{*} g+g^{*} r+\left(r^{*} g\right)^{\tau}+\left(g^{*} r\right)^{\tau}, \alpha\right\rangle=0
$$

for all $r \in \operatorname{Hom}\left(W \otimes \boldsymbol{R}^{2 n-2}, W \otimes \boldsymbol{R}^{2 n-2}\right)$. Since $\langle r, q\rangle=\left\langle r^{\tau}, q^{\tau}\right\rangle$ and $h(r q)=$ $h(q r)$, we see easily that the right hand side is $4 \mathscr{R} t r r^{*} g \alpha$. Therefore (15) is satisfied for all $r$ if and only if $g \alpha=0$. Since $\alpha$ is hermitian, it follows that the condition is equivalent to $\alpha \in \operatorname{Her}(\operatorname{Ker} g$, Ker $g$ ).

Lemma 3.5. $\operatorname{Im} \theta \cap S^{\Lambda}=\left\{\gamma-\gamma^{\tau} ; \gamma \in \operatorname{Her}(\operatorname{Ker} g, \operatorname{Ker} g)\right\}^{\perp} \cap S^{\Lambda}$.

Proof. If $\beta \in \operatorname{Image} \theta \cap S^{A}$, then for any $\gamma \in \operatorname{Her}(\operatorname{Ker} g$, Ker $g$ ),

$$
\begin{aligned}
\mathscr{R}\left\langle\beta, \gamma-\gamma^{\tau}\right\rangle & =2 \mathscr{R}\langle\beta, \gamma\rangle=2 \mathscr{R} \operatorname{tr} \gamma \beta=2 \mathscr{R} \operatorname{tr} \gamma\left(r^{*} g+g^{*} r\right) \\
& =4 \mathscr{R} \operatorname{tr}\left(r^{*} g \gamma\right)=0 .
\end{aligned}
$$

Thus the left hand side is contained in the right hand side. We prove the equality by counting the dimension of both sides. Set $\Phi=\operatorname{Her}(\operatorname{Ker} g, \operatorname{Ker} g)$ $\cap S$. Then the real dimension of the right hand side is equal to

$$
\operatorname{dim}_{R} S^{\Lambda}-\left(\operatorname{dim}_{C} \operatorname{Ker} g\right)^{2}+\operatorname{dim}_{R} \Phi .
$$

Since the left hand side is equal to the image by $\theta$ of $\operatorname{Ker} \pi_{S} \circ \theta$, its dimension is equal to

$$
\begin{aligned}
\operatorname{dim}_{R} & \left(\operatorname{Ker} \pi_{S} \circ \theta\right)-\operatorname{dim}_{R}(\operatorname{Ker} \theta) \\
= & \operatorname{dim}_{\boldsymbol{R}}\left(\operatorname{Hom}\left(W \otimes \boldsymbol{R}^{2 n-2}, W \otimes \boldsymbol{R}^{2 n-2}\right)\right)-\operatorname{dim}_{\boldsymbol{R}}\left(\operatorname{Im} \pi_{S} \circ \theta\right) \\
& \quad-\operatorname{dim}_{\boldsymbol{R}}(\operatorname{Ker} \theta)
\end{aligned}
$$




$$
\begin{aligned}
= & 2(m(2 n-2))^{2}-\left(\operatorname{dim}_{R} S-\operatorname{dim}_{R} \Phi\right)-\left((m(2 m-2))^{2}\right. \\
& +\left(\operatorname{dim}_{C}(\operatorname{Ker} g)^{2}\right) \quad(\text { by Lemma } 3.4 \text { and }(14)) \\
= & \left.(m(2 n-2))^{2}-\operatorname{dim}_{R} S\right)+\operatorname{dim}_{R} \Phi-\left(\operatorname{dim}_{C} \text { Ker } g\right)^{2} \\
= & \operatorname{dim}_{R} S^{\Lambda}-\left(\operatorname{dim}_{C} \operatorname{Ker} g\right)^{2}+\operatorname{dim}_{R} \Phi . \quad \text { q.e.d. }
\end{aligned}
$$

For $\gamma \in \operatorname{Her}\left(W \otimes \boldsymbol{R}^{2 n-2}, W \otimes \boldsymbol{R}^{2 n-2}\right)$, set

$$
C(\gamma)=\operatorname{tr}_{W}(i \gamma(I \otimes J))=\sum i c_{s t}^{0} \gamma^{s t} \in \operatorname{Hom}(W, W) .
$$

Since $J^{*}=-J, C(\gamma)$ is hermitian. If $H \in \operatorname{Her}(W, W)$, then

$$
\begin{aligned}
\langle\gamma, H \otimes J\rangle & =\operatorname{tr}(-(H \otimes J) \gamma)=i \operatorname{tr}((H \otimes I)(I \otimes J) i \gamma) \\
& =i \operatorname{tr}\left(H \operatorname{tr}_{W}(i \gamma(I \otimes J))\right)=i\langle C(\gamma), H\rangle .
\end{aligned}
$$

Thus

$$
\langle\gamma, H \otimes J\rangle=i\langle C(\gamma), H\rangle \quad(H \in \operatorname{Her}(W, W)) .
$$

Set

$$
Z=\left\{\beta \in S^{\Lambda} ; C(\beta)=0\right\} .
$$

Then by (17), $\beta \in S^{\Lambda}$ is in $Z$ if and only if $\langle\beta, H \otimes J\rangle=0$ for all $H \in \operatorname{Her}(W, W)$. Hence

$$
S^{\Lambda}=Z \oplus(\operatorname{Her}(W, W) \otimes J),
$$

where $\oplus$ indicates an orthogonal decomposition.

Lemma 3.6. Set $G=\left\{\gamma-\gamma^{\tau} ; \gamma \in \operatorname{Her}(\operatorname{Ker} g\right.$, Ker $\left.g)\right\}$. Then

$$
G=\rho_{G} Z \oplus(G \cap(\operatorname{Her}(W, W) \otimes J)) .
$$

Proof. By (19), $Z$ is orthogonal to $\operatorname{Her}(W, W) \otimes J$, so that $\rho_{G} Z$ is orthogonal to $G \cap(\operatorname{Her}(W, W) \otimes J)$. Let $v \in G$ be orthogonal to $\rho_{G} Z$. Then $v$ is orthogonal to $Z$. Since $v$ is in $S^{A}$, it is in $\operatorname{Her}(W \otimes W) \otimes J$ by (19) and hence in $G \cap(\operatorname{Her}(W, W) \otimes J)$. q.e.d.

Proposition 3.2. Assume that not all $c_{s t}^{0}$ are zero, and define $\mathscr{L} \subseteq$ Her $(W, W)$ by

$$
\mathscr{L} \otimes J=\left\{\gamma-\gamma^{\tau} ; \gamma \in \operatorname{Her}(\operatorname{Ker} g, \operatorname{Ker} g)\right\} \cap(\operatorname{Her}(W, W) \otimes J) .
$$

Then conditions (i), (ii), (iii) in Lemma 3.2 are satisfied if and only if there is a positive definite hermitian form on $W$ orthogonal to $\mathscr{L}$.

Proof. Assume that $\beta \in S^{\Lambda}$ satisfies conditions 1) and 2) in Proposition 3.1. By 1), $\left\langle\beta, \gamma^{\tau}\right\rangle=\left\langle\beta^{\tau}, \gamma\right\rangle=-\langle\beta, \gamma\rangle=-\operatorname{tr} \gamma \beta=0$ for all $\gamma \in \operatorname{Her}(\operatorname{Ker} g$, Ker $g$ ), 
so that $\beta$ is orthogonal to $G$. Thus for $H \in \mathscr{L},\langle C(\beta), H\rangle=-i\langle\beta, H \otimes J\rangle=0$ (cf. (17)). Hence $C(\beta)=\operatorname{tr}_{W}(i \beta \circ(I \otimes J))$ is orthogonal to $\mathscr{L}$ and is positive definite by 2). Conversely, assume that $h$ is a positive definite hermitian form on $W$ and is orthogonal to $\mathscr{L}$. Take $\beta_{1} \in S^{\Lambda}$ such that $C\left(\beta_{1}\right)=h$. Then $\left\langle\beta_{1}, \mathscr{L} \otimes J\right\rangle=i\left\langle C\left(\beta_{1}\right), \mathscr{L}\right\rangle=0$, so that $\beta_{1}$ is orthogonal to $G \cap(\operatorname{Her}(W, W)$ $\otimes J)$. Thus by Lemma 3.5, $\beta_{1} \in G^{\perp}+\rho_{G} Z$, where $G^{\perp}$ is the orthogonal complement of $G$ in $S^{\Lambda}$. Since $\rho_{G} Z \subset Z+G^{\perp}$, it follows that $\beta_{1} \in G^{\perp}+Z$. Write $\beta_{1}=\beta+\zeta$, where $\beta \in G^{\perp}$ and $\zeta \in Z$. Then $C(\beta)=C\left(\beta_{1}\right)-C(\zeta)=C\left(\beta_{1}\right)=h$. Thus $C(\beta)>0$. Since $\beta \in G^{\perp}$, for any $\gamma \in \operatorname{Her}(\operatorname{Ker} g$, Ker $g$ ) we have $\langle\beta, \gamma\rangle=$ $\frac{1}{2}\left\langle\beta, \gamma-\gamma^{\tau}\right\rangle=0$. Thus $\operatorname{tr} \beta \gamma=0$ for all $\gamma \in \operatorname{Her}(\operatorname{Ker} g$, Ker $g)$, and hence $\beta$ satisfies condition 2) in Proposition 3.1. q.e.d.

By considering the conditions in Lemma 3.2 for $d=2$, we obtain a more general condition for half-estimate. We can write down these conditions parallel to Proposition 3.1 as follows:

Proposition 3.3. Conditions (i,) (ii) $)_{2}$, (iii) $)_{2}$ in Lemma 3.2 are satisfied if and only if there is $\beta \in S^{4}$ such that

1) $\rho_{K} \beta \rho_{K} \geq 0 \quad$ where $K=\operatorname{ker} g$,

2) $\operatorname{tr}_{W}(i \beta(I \otimes J))>0$.

Proof. Assume that $g_{\theta}$ satisfies (i), (ii) $)_{2}$, and (iii) $)_{3}$, Write

$$
g_{\theta}=g+\theta r+\theta^{2} q \quad\left(\bmod \theta^{3}\right) .
$$

Then (ii) ${ }_{2}$ and (iii) ${ }_{2}$ are equivalent to

$$
\begin{gathered}
g^{*} r+r^{*} g+\left(g^{*} r+r^{*} g\right)^{\tau}=0, \\
g^{*} q+q^{*} g+r^{*} r+\left(g^{*} q+q^{*} g+r^{*} r\right)^{\tau}=0 \\
\left\langle i t r_{W}\left(\theta\left(g^{*} r+r^{*} g\right)+\theta^{2}\left(g^{*} q+q^{*} g+r^{*} r\right)\right)(I \otimes J) u, u\right\rangle \geq c \theta^{2}|u|^{2}
\end{gathered}
$$

for all sufficiently small $\theta$ and all $u \in W$. Set

$$
H_{1}=i t r_{W}\left(\left(g^{*} r+r^{*} g\right)(I \otimes J)\right), H=i t r_{W}\left(\left(g^{*} q+q^{*} g+r^{*} r\right)(I \otimes J)\right) .
$$

(22) implies that for a sufficiently large real number $a, a H_{1}+H_{2}>0$. Set

$$
f=q+a r, \quad \beta=g^{*} f+f^{*} g+r^{*} r,
$$

Then $\operatorname{tr}_{W}(i \beta(I \otimes J))>0$ by (22), and $\beta \in S^{\Lambda}$ by (20) and (21). Moreover, $\rho_{K} \beta \rho_{K}=\rho_{K} r^{*} r \rho_{K} \geq 0$. Thus $\beta$ satisfies our conditions. Conversely, assume that there is $\beta \in S^{\Lambda}$ satisfying our conditions. Write $\rho_{K} \beta \rho_{K}=r^{*} r$, where $r \in \operatorname{Hom}(\operatorname{Ker} g, \operatorname{Ker} g)$. Then $\rho_{K}\left(\beta-r^{*} r\right) \rho_{K}=0$, and therefore there is $q \in \operatorname{Hom}\left(W \otimes \boldsymbol{R}^{2 n-2}, W \otimes \boldsymbol{R}^{2 n-2}\right)$ such that 


$$
\beta-r^{*} r=g^{*} q+q^{*} g .
$$

Noting $r^{*} g=g^{*} r=0$ since $\operatorname{Im} g \perp$ Ker $g$, we see easily that $g_{\theta}=g+\theta r+$ $\theta^{2} q$ satisfies our requirements (20), (21) and (22).

\section{Appendix}

Proof of Theorem 1.4. Set $\langle\xi\rangle=\left(1+|\xi|^{2}\right)^{\frac{1}{2}}$. Expanding $J\left(x, \xi+z\langle\xi\rangle^{\frac{1}{2}}\right)$ in Taylor's series in $z$, multiplying by $b t g(z)^{2}$, integrating over $\boldsymbol{R}^{n}$ in $z$, and noting that $g(z)$ is an even function, we find that

$$
J(x, \xi)=J_{1}(x, \xi)-\left(\partial^{2} J(x, \xi) / \partial \xi_{j} \partial \xi_{k}\right) a_{j k}\langle\xi\rangle+R(x, \xi),
$$

where

$$
J_{1}(x, \xi)=\int J\left(x, \xi+z\langle\xi\rangle^{\frac{1}{2}}\right) g(z)^{2} d z,
$$

and $R(x, \xi)$ is of order $l-2$. Set

$$
r(\chi, \xi)=\hat{J}(\chi, \xi), \quad \gamma_{1}(\chi, \xi)=\hat{J}_{1}(\chi, \xi),
$$

where $\wedge$ indicates Fourier transform in the space variables. Then by applying a change of variables $z=\langle\xi\rangle^{-\frac{1}{2}}(\zeta-\xi)$ to (13),

$$
\gamma_{1}(\chi, \xi)=\int \gamma(\chi, \xi) g\left(\langle\xi\rangle^{-\frac{1}{2}}(\zeta-\xi)\right)^{2}\langle\xi\rangle^{-n / 2} d \zeta .
$$

In view of (13) we are interested in estimating $\int\left\langle\gamma_{1}(\chi-\xi) \hat{u}(\xi), \hat{u}(\chi)\right\rangle d \xi d \chi$ from below. However, instead of $\gamma_{1}$ we first consider

$$
\begin{gathered}
\gamma_{2}(\chi, \xi)=\int \gamma(\chi, \zeta) g\left(\langle\xi+\chi\rangle^{-\frac{1}{2}}(\zeta-\xi-\chi)\right)\langle\xi+\chi\rangle^{-n / 4} \\
\cdot g\left(\langle\xi\rangle^{-\frac{1}{2}}(\zeta-\xi)\right)\langle\xi\rangle^{-n / 4} d \zeta \\
=\int \gamma\left(\chi, \xi+z\langle\xi\rangle^{\frac{1}{2}}\right) g\left(\langle\xi+\chi\rangle^{-\frac{1}{2}}\left(z\langle\xi\rangle^{\frac{1}{2}}-\chi\right)\right) \\
\cdot\langle\xi+\chi\rangle^{-n / 4} g(z)\langle\xi\rangle^{n / 4} d z,
\end{gathered}
$$

and then study the difference $\gamma_{1}-\gamma_{2}$.

From the first defining formula of $\gamma_{2}(\chi, \xi)$, it follows that

$$
\int\left\langle r_{2}(\chi-\xi, \xi) \hat{u}(\xi), \hat{u}(\chi)\right\rangle d \xi d \chi=\int\left\langle J(x, \zeta) u_{\zeta}(x), u_{\zeta}(x)\right\rangle d x d \zeta,
$$

where $\hat{u}_{\xi}(\xi)=g\left(\langle\xi\rangle^{-\frac{1}{2}}(\zeta-\xi)\right)\langle\xi\rangle^{-n / 4} \hat{u}(\xi)$. Therefore by our assumption, 


$$
\mathscr{R} \int\left\langle\gamma_{2}(\chi-\xi, \xi) \hat{u}(\xi), \hat{u}(\chi)\right\rangle d \xi d \chi \geq 0
$$

By (2) and the second defining formula of $\gamma_{2}(\chi, \xi)$ in (17),

$$
\begin{aligned}
& \gamma_{1}(\chi, \xi)-\gamma_{2}(\chi, \xi)=\int \gamma\left(\chi, \xi+z\langle\xi\rangle^{\frac{1}{2}}\right) \\
& \quad \cdot\left\{g\left(\langle\xi+\chi\rangle^{-\frac{1}{2}}\left(z\langle\xi\rangle^{\frac{1}{2}}-\chi\right)\right)\langle\xi+\chi\rangle^{-n / 4}\langle\xi\rangle^{n / 4}-g(z)\right\} g(z) d z .
\end{aligned}
$$

Note that

$$
\begin{gathered}
\langle\xi+\chi\rangle^{-a}\langle\xi\rangle^{a}=1-a\langle\xi\rangle^{-2}(\xi, \chi)+R_{a}(\chi, \xi), \\
\left|R_{a}(\chi, \xi)\right| \leq C_{a}\langle\xi\rangle^{-2}\langle\chi\rangle^{|a+2|+4},
\end{gathered}
$$

where $(\xi, \chi)$ is the inner product of $\xi$ and $\chi$. Therefore

$$
\begin{gathered}
g\left(\langle\xi+\chi\rangle^{-\frac{1}{2}}\left(z\langle\xi\rangle^{\frac{1}{2}}-\chi\right)\right)\langle\xi+\chi\rangle^{-n / 4}\langle\xi\rangle^{n / 4}-g(z) \\
=-(n / 4)\langle\xi\rangle^{-2}(\xi, \chi) g(z)-\left(\frac{1}{2}\langle\xi\rangle^{-2}(\xi, \chi) z_{j}+\langle\xi\rangle^{-\frac{1}{2}} \chi_{j}\right) \partial g / \partial z_{j} \\
+\frac{1}{2}\langle\xi\rangle^{-1} \chi_{j} \chi_{k} \partial^{2} g / \partial z_{j} \partial z_{k}+S_{1}(\chi, \xi, z), \\
\left|S_{1}(\chi, \xi, z)\right| \leq C\langle\xi\rangle^{-3 / 2}\langle\chi\rangle^{k}\langle z\rangle^{k}
\end{gathered}
$$

for a sufficiently large $k$. Since

$$
\begin{gathered}
\gamma\left(\chi, \xi+z\langle\xi\rangle^{\frac{1}{2}}\right)=\gamma(\chi, \xi)+z\langle\xi\rangle^{\frac{1}{2}} \partial \gamma(\chi, \xi) / \partial \xi_{\nu}+S_{2}(\chi, \xi, z), \\
\left|S_{2}(\chi, \xi, z)\right| \leq C_{N}\langle\xi\rangle^{-1}\langle z\rangle^{k}\langle\chi\rangle^{-N}
\end{gathered}
$$

it follows then by (20) that

$$
\begin{aligned}
\gamma_{1}(\chi, \xi)-\gamma_{2}(\chi, \xi)= & \frac{1}{2} \gamma(\chi, \xi)\langle\xi\rangle^{-1} \chi_{j} \chi_{k} \int\left(\partial^{2} g / \partial z_{j} \partial z_{k}\right) g(z) d z \\
& -\gamma(\chi, \xi)\langle\xi\rangle^{-2}(\xi, \chi)\left\{(n / 4)+\frac{1}{2} \int z_{j} g(z)\left(\partial g / \partial z_{j}\right) d z\right\} \\
& -\chi_{j}\left(\partial \gamma(\chi, \xi) / \partial \xi_{k}\right) \int z_{k} g(z)\left(\partial g / \partial z_{j}\right) d z+S(\chi, \xi) \\
= & \frac{1}{2} \gamma(\chi, \xi)\langle\xi\rangle^{-1} \chi_{j} \chi_{k} b_{j k}+\hat{T}(\chi, \xi)+S(\chi, \xi)
\end{aligned}
$$

where $|S(\chi, \xi)| \leq C_{N}\langle\xi\rangle^{l-3 / 2}\langle\chi\rangle^{-N}, T(x, \xi)$ is of order $l-1$, and

$$
\mathscr{R}\langle T(x, \xi) u, u\rangle=0
$$

for all $u$. The above equations together with (13), (18) and (19) therefore give $\mathscr{R}\langle J(x, D) u, u\rangle \geq-\mathscr{R}\langle L(x, D) u, u\rangle+\mathscr{R}\langle T(x, D) u, u\rangle+\mathscr{R}\langle S(x, D) u, u\rangle$. 
Since $\mathscr{R}\langle T(x, D) u, u\rangle=0$ we can apply our argument to $\langle T(x, D) u, u\rangle$. Note that we have not used the hermitian assumption of $J(x, \xi)$ except for getting (21). Since $T(x, \xi)$ is of order $l-1$ we find that $|\mathscr{R}\langle T(x, D) u, u\rangle| \geq$ $+\mathscr{R}\left\langle S^{\prime}(x, D) u, u\right\rangle$ where $S^{\prime}(x, \xi)$ is of order $\leq l-2$. This completes the proof of our theorem.

\section{References}

[1] A. P. Calderón, Boundary value problems for elliptic equations, Outlines SovietAmerican Sympos. Partial Differential Equations, Novosibirsk, 1963, 303-304.

[2] K. O. Friedrichs, Pseudo-differential operators, Lecture notes, Courant Inst. Math. Sci., New York University, 1968.

[ 3 ] L. Hörmander, Pseudo-differential operators and non-elliptic boundary problems, Ann. of Math. 83 (1966) 129-209.

[4] - Pseudo-differential operators and hypoelliptic equations, Proc. Sympos. Pure Math. Vol. 10, Amer. Math. Soc., 1967, 138-183.

[ 5 ] J. J. Kohn, Harmonic integrals on strongly pseudo-convex manifolds. I, II, Ann. of Math. 78 (1963) 112-145, 79 (1964) 450-472.

[6] - Boundaries of complex manifolds, Proc. Conf. Complex Analysis (Minneapolis), Springer, Berlin, 1965, 81-94.

[7] J. J. Kohn \& L. Nirenberg, An algebra of pseudo-differential operators, Comm. Pure Appl. Math. 18 (1965) 269-305.

[8] - Non-coercive boundary value problems, Comm. Pure Appl. Math. 18 (1965) 443-492.

[9] C. B. Morrey, The analytic embedding of abstract real-analytic manifolds, Ann. of Math. 68 (1958) 159-201.

[10] R. T. Seeley, Singular integrals and boundary value problems, Amer. J. Math. 88 (1966) 781-809.

[11] D. C. Spencer, Overdetermined systems of linear partial differential equations, Bull. Amer. Math. Soc. 75 (1969) 179-239.

[12] W. J. Sweeney, A non-compact Dirichlet norm, Proc. Nat. Acad. Sci. U.S.A. 58 (1967) 2193-2195.

Columbia University 
